\title{
OPEN-CHANNEL MICROFLUIDICS: FREE-STANDING HYDROGEL MICROARRAYS FOR PROTEIN ELECTROPHORESIS
}

\author{
T.A. Duncombe ${ }^{1}$, T.M. Tran ${ }^{1}$, F. Benito-Lopez ${ }^{2}$, D. Diamond ${ }^{2}$, and A.E. Herr 1,3* \\ ${ }^{1}$ UC Berkeley / UC San Francisco Joint Graduate Group in Bioengineering, USA \\ ${ }^{2}$ CLARITY: Centre for Sensor Web Technologies, National Centre for Sensor Research, School of Chemical \\ Sciences, Dublin City University, Dublin 9, IRELAND \\ ${ }^{3}$ University of California, Berkeley, CA 94720, USA
}

\begin{abstract}
We report a new open-channel architecture that enables the utility of microfluidics without sacrificing accessibility for high performance protein electrophoresis. We utilize photo-patterned free-standing polyacrylamide gels as both an open-microchannel and a sieving matrix for protein sizing. The open format allows for easy downstream sample access for immunoblotting after rapid microfluidic protein separations are performed. For the purposes of multiplexing, the assay footprint is minimized to a single channel through the implementation of moving boundary electrophoresis protein separations.
\end{abstract}

\section{INTRODUCTION}

Contemporary microfluidic technologies are realized as miniaturized platforms comprising enclosed microchannels to exploit the unique physical attributes of the microscale. In microfluidic electrochromatography, the high surface area to volume ratio results in efficient heat dissipation enabling the application of high electric fields for rapid separations. To date, a principle limitation of microfluidics in closed channel formats is the inability for extraction and downstream processing of the sample. While digital microfluidics (e.g., EWOD) has remedied this for discrete droplet reactors [1], such advances have been attempted [2] but have not matured in separation science.

Consequently, we report here for the first time a new open-channel architecture that enables the utility of microfluidics without sacrificing accessibility - for high performance protein electrophoresis. Owing to its open architecture, this technology is optimized for interfacing with automated robotic controllers and downstream processing (i.e., sample spotters, immunological detection, mass spectroscopy). The unmatched accessibility makes this platform uniquely well-suited to massively parallelized proteomics, a major unrealized goal from bioanalytical technology.

In a notable departure from protein micro-separations approaches [3-6], the present study introduces a unique open-channel format for protein electrophoresis (Figure 1). Here we demonstrate a photo-patterning technique to realize free-standing polyacrylamide gel microchannels. A protein separation is demonstrated in a single free-standing microchannel by moving boundary electrophoresis (MBE). In MBE the moving boundary of analytes are analyzed, as opposed to discrete zones, thus eliminating the need for an injection channel. Polyacrylamide gel MBE enables rapid protein separations in short single channels [3]. The MBE format allows separations to be realized on a small device footprint and with low power consumption, both important when implementing a multiplexed array.

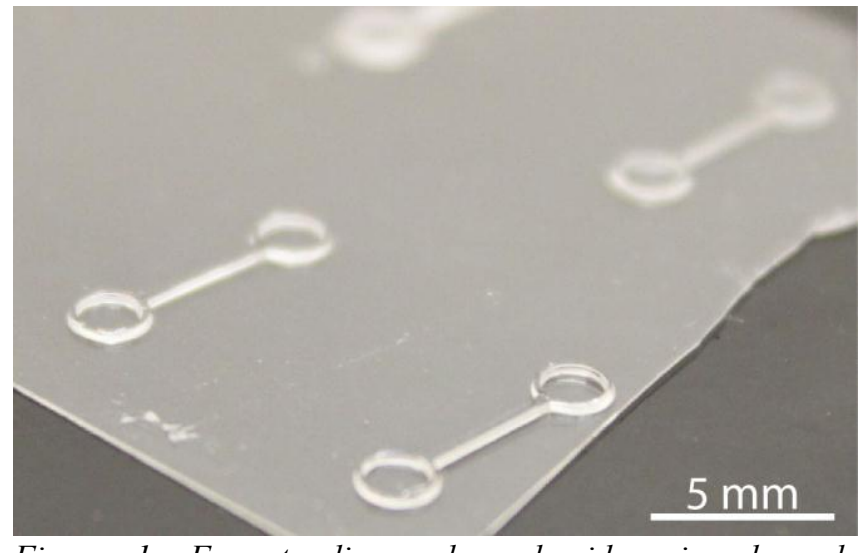

Figure 1: Free-standing polyacrylamide microchannels arrays are a fully accessible microfluidic platform easily interfaced with automated robotic fluid handling and operation.

\section{MATERIALS AND METHODS \\ Materials}

Solutions of 30\% (29:1) acrylamide/bis-acrylamide, 3(trimethoxysilyl)-propyl methacrylate $(98 \%)$, glacial acetic acid, methanol and glass coverslips were purchased from Sigma Aldrich (St. Louis, MO). Photoinitiator 2,2-azobis[2methyl-N-(2-hydroxyethyl) propionamide] (VA-086) was purchased from Wako Chemical (Richmond, VA). Alexa Fluor 488 conjugated Trypsin Inhibitor (TI), Ovalubmin (OVA), and Bovine Serum Albumin (BSA) were purchased from Sigma. Tris-glycine (10x) native electrophoresis buffer was purchased from Bio-Rad Laboratories (Hercules, CA).

\section{Coverslip Functionalization}

A glass coverslip is cleaned in a $1 \mathrm{M} \mathrm{NaOH}$ solution for 30 minutes and then washed with DI water and dried with nitrogen. A 20 L drop of 2:3:5 (v/v/v) mixture of 3(trimethoxysilyl)-propyl methacrylate, glacial acetic acid and DI water is sandwiched between a petri dish and a cleaned coverslip. The petri dish is sealed and placed in refrigerator at $4^{\circ} \mathrm{C}$ overnight. Afterwards the coverslip is rinsed with methanol and DI water and stored dry until it is used. 

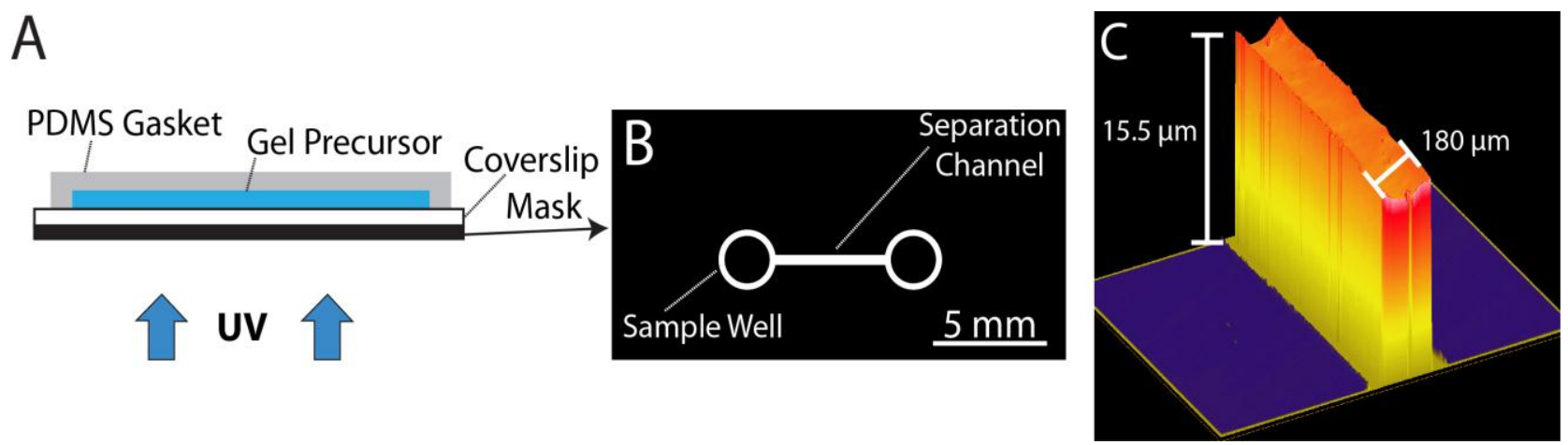

Figure 2: Free-standing polyacrylamide gel microchannels are fabricated using a (A) mask based photolithography technique. A reservoir of gel precursor is contained above a methacrylate functionalized coverslip. The desired micropattern is defined by a photo-mask, which determines the portions of the reservoir that are polymerized by a UV light source. Our design (B) consists of a single channel connecting two fluid reservoirs. (C) Optical profilometry shows well defined free-standing hydrogel with dimensions and uniformity appropriate for supporting electrokinetic protein separations.

\section{Free-Standing Gel Fabrication}

Gel precursor consisted of a $10 \%(\mathrm{w} / \mathrm{v})$ acrylamide concentration with a bis-acrylamide crosslinker ratio of $3 \%$ $(\mathrm{w} / \mathrm{w})$, and 1\% (w/v) VA-086 dissolved in DI water. After degassing, the precursor solution is placed in a polydimethylsiloxane gasket atop a glass coverslip that has been functionalized with methacrylate (Figure 2A). A BlakRay ${ }^{\circledR}$ UV lamp at $10 \mathrm{~mW} / \mathrm{cm}^{2}$ is exposed through a Mylar photo-mask (Figure 2B) on the back side of the coverslip for 5 minutes. After polymerization, the gasket is removed and excess precursor is washed away leaving only the freestanding gel microstructure behind. We establish fluid reservoirs by fabricating circular rings of gel, with the capacity to hold 2 L of sample delivered with a pipette.

Optical profilometry is performed to confirm a high level of controllability in the free-standing structure with a channel cross section of $180 \mathrm{~m}$ wide by $15.5 \mathrm{~m}$ tall in its hydrated state (Figure 2C). The gels are dehydrated for storage. They can be rehydrated in any aqueous buffer of interest and ready for experimentation in minutes.

\section{EXPERIMENTAL}

\section{Environmental Chamber}

A key hurdle to realizing electrokinetic separations in the open-channel format is evaporation. In ambient conditions we observed rapid evaporation that resulted in unsteady electrical current over the course of the separation. To alleviate the problem we utilized a small environmental chamber, consisting of an inverted petri dish and a moist KIMWIPE $^{\circledR}$. Using this technique a stable electrical current is established.

\section{Testing Procedure}

Free-standing gels were rehydrated in a large droplet of $1 \mathrm{x}$ tris/glycine for 5 minutes. After gel hydration the excess buffer was removed from gel using a KIMWIPE ${ }^{\circledR}$. The sample of interest is pipetted into the sample well and a run buffer is pipetted in to the opposite, sample wash well.
Platinum electrodes are aligned and inserted from above into the two wells and electrophoresis is immediately initiated by a Caliper high voltage power supply.

Protein fronts are visualized using an inverted epiflourescence microscope (Olympus IX-70) equipped with a $100 \mathrm{~V}$ mercury arc lamp, a 10x objective, and a Peltiercooled charge-coupled device (CCD) camera (CoolSNAP HQ2, Roper Scientific, Trenton, NJ). Images are recorded using MetaMorph ${ }^{\circledR}$ acquisition software and post-processing was done in Image $(\mathrm{NIH})$ and $\mathrm{MATLAB}^{\circledR}$.

\section{Separation Efficiency}

The ability to resolve two analytes is quantified through 'separation resolution' (SR, the mean distance between neighboring peaks normalized by the average peak width). A $\mathrm{SR}>1$ indicates a successful separation. In this study we monitor the 'critical separation length', or the migration distance required to realize a $\mathrm{SR}>1$. As the critical separation length is reduced, so does the required channel length.

\section{RESULTS}

A protein separation was performed in the free-standing polyacrylamide gel format and is displayed in Figure 3. A fluorescently labeled sample of $250 \mathrm{~nm}$ BSA, $250 \mathrm{~nm}$ OVA, and $250 \mathrm{~nm}$ TI in $1 \mathrm{x}$ tris/glycine is baseline resolved in 2 minutes and in the first $250 \mathrm{~m}$ of migration. In Figure $3 \mathrm{~A}$ the protein moving boundaries are clearly visible several millimeters along the separation channel. Figure 3B displays the first $250 \mathrm{~m}$ of migration at the 130 seconds into the separation. The image is false colored such that the TI, OVA, and BSA fronts can be clearly distinguished. The corresponding fluorescence intensity plot is shown in Figure $3 \mathrm{C}$ and is overlaid with the derivative of the intensity over location $(\mathrm{dI} / \mathrm{dx})$. In the $\mathrm{dI} / \mathrm{dx}$ plot the unique protein species can be clearly identified. 

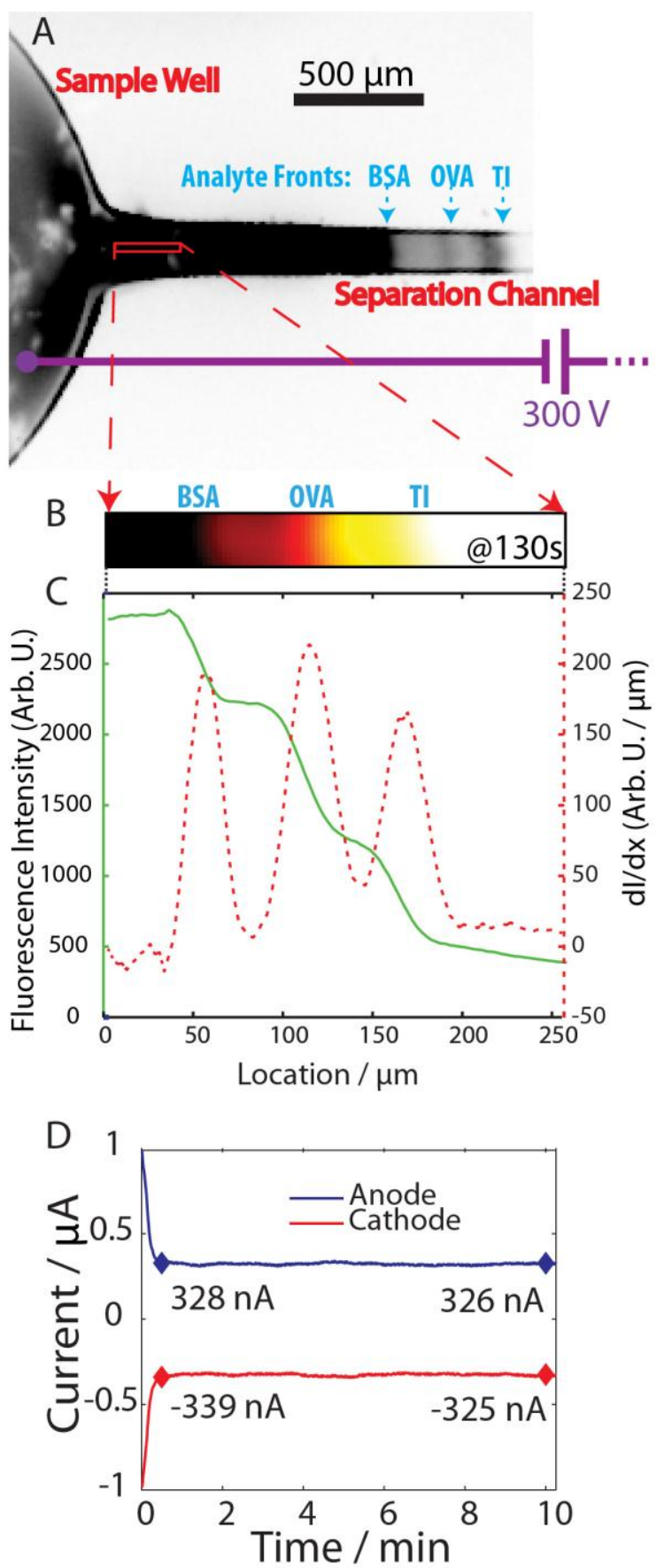

Figure 3: A free-standing 10\% (w/v) acrylamide gel acts as both the microchannel and sieving matrix for a native $M B E$ separation (A). 250 nM fluorescently labeled protein ladder consisting of Trypsin Inhibitor (TI), Ovalbumin (OVA) and Bovine Serum Albumin (BSA) is captured in an epifluorescence image. (B) A false color image of the first 250 $\mu m$ of the separation channel at 130s clearly shows well separated proteins, a $2.7 x$ reduction in separation length as compared to on-chip [3]. The corresponding (C) intensity profile and the derivative of the front $d I / d x$ are overlaid. (D) The environmental chamber prevents appreciable current reduction.

To visualize the separation in location and time, $\mathrm{dI} / \mathrm{dx}$ is plotted at 10 second intervals along the first $250 \mathrm{~m}$ of migration in Figure 4A. As the separation progresses in time and location the resolution of proteins improves. An automated Matlab Gaussian curve fitting program is used to determine the mean location and the dispersion of the protein species. The separation resolution is plotted as a function of time in Figure 4B. All three separations surpass the critical SR in under 50 seconds as compared to hours that would be required for a conventional benchtop slab-gel separation.
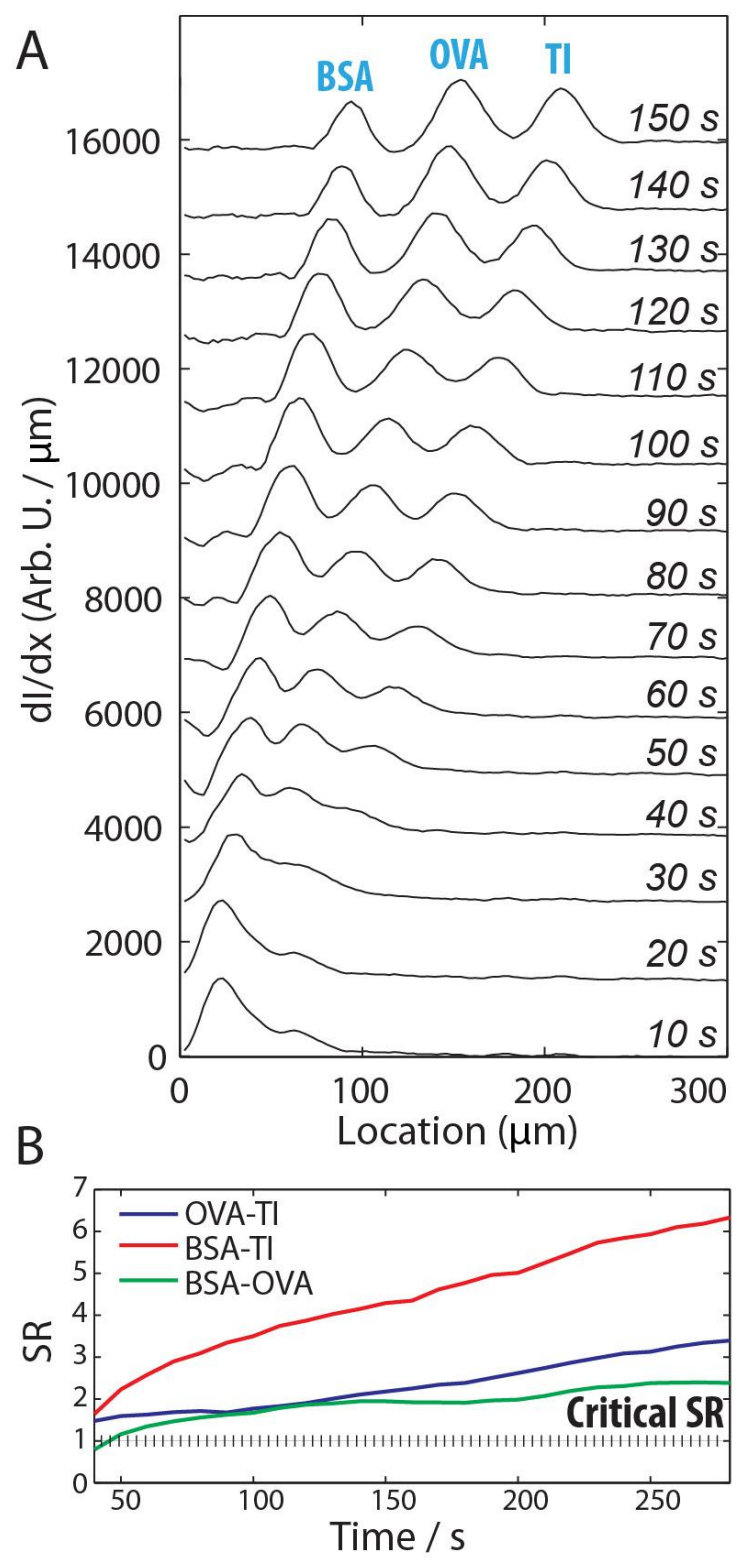

Figure 4: The MBE free-standing gel separation of a three protein ladder is completed in 50s and in the first $150 \mu \mathrm{m}$. (A) $d I / d x$ is staggered in time to visualize the protein migration with the $(B)$ separation resolution plotted for each protein separation from the Figure 3 experiment. 


\section{DISCUSSION}

We introduce a novel free standing polyacrylamide gel microarray platform for protein electrophoresis. Each microgel acts as both a microchannel and a sieving matrix for rapid protein analysis. The accessible microfluidic format is appropriate for massive scale up into large arrays, offering facile integration with automated robotics for highthroughput proteomics.

\section{ACKNOWLEDGEMENTS}

The authors gratefully acknowledge members of The Herr Laboratory at UC Berkeley for assistance and helpful discussions. The authors acknowledge financial support from the US National Science Foundation (NSF) in the form of both Graduate Research Fellowships (TAD, TMT) and CAREER Award (AEH, grant \# 1056035). Additional support was provided by the Science Foundation of Ireland (FBL, DD, grant 07/CE/I1147) and the Short Term Travel Fellowship Supplement 2011 (FBL). Partial conference travel support was provided by the Transducer Research Foundation. Partial infrastructure support was provided by the UC Berkeley/QB3 Biomolecular Nanofabrication Center (BNC). A.E.H. is an Alfred P. Sloan research fellow in chemistry.

\section{REFERENCES}

[1] Abdelgawad, M.; Freire, S. L. S.; Yang, H.; Wheeler, A. R., All-terrain droplet actuation. Lab on a Chip 2008, 8, 672-677.

[2] Liu, J.; Tseng, K.; Garcia, B.; Lebrilla, C. B.; Mukerjee, E.; Collins, S.; Smith, R., Electrophoresis Separation in Open Microchannels. A Method for Coupling Electrophoresis with MALDI-MS. Analytical Chemistry 2001, 73 (9), 2147-2151.

[3] Duncombe, T. A.; Tentori, A. M.; Hou, C.; Herr, A. E., From Bench-To-Bedside: Realizing On-Chip Electrophoretic Immunoassays For Protein Biomarkers In Serum Using a Standard 9V Battery. In IEEE MEMS Conference, 2012.

[4] Apori, A. A.; Herr, A. E., Homogeneous immunosubtraction integrated with sample preparation enabled by a microfluidic format. Anal Chem 2011, 83 (7), 2691-8

[5] Hughes, A. J.; Herr, A. E., Quantitative enzyme activity determination with zeptomole sensitivity by microfluidic gradient-gel zymography. Anal Chem 2010, 82 (9), 3803-11.

[6] Hou, C.; Herr, A. E., Ultrashort separation length homogeneous electrophoretic immunoassays using onchip discontinuous polyacrylamide gels. Anal Chem 2010, 82 (8), 3343-51.

[7] Khetan, S.; Burdick, J. A., Patterning hydrogels in three dimensions towards controlling cellular interactions. Soft Matter 2011, 7 (3).

\section{CONTACT}

*A.E. Herr, tel: +1-510-666-3396; aeh@berkeley.ed 